\title{
Genetic risk for atherothrombotic cerebral infarction in individuals stratified by sex or conventional risk factors for atherosclerosis
}

\author{
SACHIYO YAMAGUCHI ${ }^{1}$, YOSHIJI YAMADA ${ }^{1,2}$, NORIFUMI METOKI ${ }^{3}$, HIDEMI YOSHIDA $^{4}$, \\ KEI SATOH ${ }^{4}$, SAHOKO ICHIHARA ${ }^{1}$, KIMIHIKO KATO ${ }^{5}$, TAKASHI KAMEYAMA ${ }^{5}$, KIYOSHI YOKOI ${ }^{5}$, \\ HITOSHI MATSUO ${ }^{6}$, TOMONORI SEGAWA ${ }^{6}$, SACHIRO WATANABE ${ }^{6}$ and YOSHINORI NOZAWA ${ }^{2}$ \\ ${ }^{1}$ Department of Human Functional Genomics, Life Science Research Center, Mie University, Tsu; \\ ${ }^{2}$ Gifu International Institute of Biotechnology, Kakamigahara, Japan; ${ }^{3}$ Department of Internal Medicine, \\ Hirosaki Stroke Center, Hirosaki; ${ }^{4}$ Department of Vascular Biology, Institute of Brain Science,
}

Hirosaki University School of Medicine, Hirosaki; ${ }^{5}$ Department of Cardiovascular Medicine and Department of Neurology, Gifu Prefectural Tajimi Hospital, Tajimi; ${ }^{6}$ Department of Cardiology, Gifu Prefectural Gifu Hospital, Gifu, Japan

Received May 22, 2006; Accepted July 20, 2006

\begin{abstract}
The aim of the present study was to assess the genetic risk for atherothrombotic cerebral infarction (ACI) in men and women separately as well as in individuals with or without conventional risk factors for atherosclerosis and thereby to contribute to the personalized prevention of ACI. The study population comprised 2705 unrelated Japanese individuals (1244 men, 1461 women), including 636 subjects (372 men, 264 women) with ACI. Subjects with ACI and controls either had or did not have conventional risk factors for atherosclerosis, including hypertension, hypercholesterolemia, and diabetes mellitus. The genotypes for 202 polymorphisms of 152 candidate genes were determined by a method that combines polymerase chain reaction and sequence-specific oligonucleotide probes with suspension array technology. Multivariable logistic regression analysis and a stepwise forward selection procedure revealed that 11 different polymorphisms were significantly $(\mathrm{P}<0.005)$ associated with ACI in women or men or in individuals with or without hypertension, hypercholesterolemia, or diabetes mellitus: the $584 \mathrm{C} \rightarrow \mathrm{T}$ polymorphism of $L I P G, 5665 \mathrm{G} \rightarrow \mathrm{T}$ of $E D N 1$, and $\mathrm{G} \rightarrow \mathrm{A}$ of $C C L 11$ in women; $677 \mathrm{C} \rightarrow \mathrm{T}$ of $M T H F R, 1323 \mathrm{C} \rightarrow \mathrm{T}$ of $I T G B 2,3932 \mathrm{~T} \rightarrow \mathrm{C}$ of $A P O E$, and $-231 \mathrm{~A} \rightarrow \mathrm{G}$ of $E D N R A$ in men; $-572 \mathrm{G} \rightarrow \mathrm{C}$ of IL6 in hypertensive individuals; $-403 \mathrm{G} \rightarrow \mathrm{A}$ of $C C L 5$ and $\mathrm{G} \rightarrow \mathrm{A}$ of $C O M T$ in individuals with hypercholesterolemia; and 3932T $\rightarrow \mathrm{C}$ of APOE and $\mathrm{A} \rightarrow \mathrm{G}$ of TNFSF4 in
\end{abstract}

Correspondence to: Dr Yoshiji Yamada, Department of Human Functional Genomics, Life Science Research Center, Mie University, 1577 Kurima-machiya, Tsu, Mie 514-8507, Japan

E-mail: yamada@gene.mie-u.ac.jp

Key words: polymorphism, genetics, ischemic stroke, atherothrombotic cerebral infarction, atherosclerosis, risk factor diabetic individuals. Polymorphisms associated with ACI may thus differ between women and men as well as among individuals with different risk factors. Stratification of subjects on the basis of sex or conventional risk factors for atherosclerosis may therefore be important in order to achieve the personalized prevention of ACI with the use of genetic information.

\section{Introduction}

Stroke is the third most common cause of death after cancer and heart disease and is the leading cause of acquired disability in adults (1). In the United States, approximately 700,000 individuals suffer a new or recurrent stroke and nearly 160,000 die from stroke-related causes each year. The total number of individuals who have experienced a stroke is 5.5 million (2). In Japan, the prevalence of stroke is 1.4 million, with nearly 132,000 deaths from this condition occurring each year (Ministry of Health, Labor, and Welfare of Japan).

Ischemic stroke, which accounts for approximately $80 \%$ of all strokes, is a complex disorder. The main cause of ischemic stroke is atherothrombosis, with the principal and treatable risk factors including hypertension, hypercholesterolemia, and diabetes mellitus (3). In addition to these conventional risk factors, genetic variants are important in the pathogenesis of ischemic stroke $(4,5)$. Recent genetic epidemiological studies have thus identified several genes related to the prevalence of stroke, including those for interleukin-6 $(6,7)$, methylenetetrahydrofolate reductase $(8,9)$, paraoxonase $(10)$, phosphodiesterase 4D (11), 5-lipoxygenase activating protein (12), and cyclooxygenase 2 (13). However, the genetic determinants of ischemic stroke remain largely unknown.

We hypothesized that gene polymorphisms related to atherothrombotic cerebral infarction (ACI) might differ between women and men as well as among individuals with or without conventional risk factors for atherosclerosis. We have therefore performed an association study of 202 
Table I. Characteristics of male and female subjects with ACI and controls.

\begin{tabular}{|c|c|c|c|c|c|c|}
\hline \multirow[b]{2}{*}{ Characteristic } & \multicolumn{3}{|c|}{ Women } & \multicolumn{3}{|c|}{ Men } \\
\hline & ACI & controls & $\mathrm{P}$ & ACI & controls & $\mathrm{P}$ \\
\hline No. of subjects & 264 & 1197 & & 372 & 872 & \\
\hline Age (years) & $68.6 \pm 12.2$ & $62.8 \pm 12.0$ & $<0.0001$ & $66.3 \pm 10.2$ & $61.7 \pm 12.0$ & $<0.0001$ \\
\hline BMI $\left(\mathrm{kg} / \mathrm{m}^{2}\right)$ & $23.7 \pm 3.5$ & $23.5 \pm 3.2$ & 0.3200 & $23.0 \pm 2.8$ & $23.3 \pm 2.8$ & 0.0780 \\
\hline Smoking (\%) & 1.9 & 3.2 & 0.2400 & 22.6 & 32.5 & 0.0004 \\
\hline Hypertension (\%) & 72.7 & 35.8 & $<0.0001$ & 66.9 & 45.3 & $<0.0001$ \\
\hline Hypercholesterolemia (\%) & 39.4 & 32.3 & 0.0275 & 30.9 & 23.1 & 0.0068 \\
\hline Diabetes mellitus (\%) & 33.7 & 15.9 & $<0.0001$ & 42.7 & 22.4 & $<0.0001$ \\
\hline
\end{tabular}

Data for age and BMI are means \pm SD.

polymorphisms and ACI for women and men separately as well as for individuals with or without hypertension, hypercholesterolemia, or diabetes mellitus. Our aim was to assess the genetic risk for ACI in men and women as well as in individuals with or without conventional risk factors for atherosclerosis and thereby to contribute to the personalized prevention of ACI.

\section{Materials and methods}

Study population. The study population comprised 2705 unrelated Japanese individuals (1244 men, 1461 women) who either visited outpatient clinics of or were admitted to one of the participating hospitals (Gifu Prefectural Gifu, Tajimi, and Gero Hot Spring Hospitals; Hirosaki University Hospital; Reimeikyo Rehabilitation Hospital; and Yokohama General Hospital) between October 2002 and March 2005. A total of 636 consecutive subjects ( 372 men, 264 women) with ACI was enrolled in the study. The diagnosis of ischemic stroke was based on the occurrence of a new and abrupt focal neurological deficit, with neurological symptoms and signs persisting for more than $24 \mathrm{~h}$; it was confirmed by positive findings in computed tomography or magnetic resonance imaging (or both) of the head. The type of stroke was determined according to the Classification of Cerebrovascular Diseases III (14). Individuals with cardiogenic embolic infarction, lacunar infarction, transient ischemic attack, hemorrhagic stroke, cerebrovascular malformations, brain tumors, or traumatic cerebrovascular diseases were excluded from the study, as were those with atrial fibrillation in the absence or presence of valvular heart disease.

The 2069 control subjects ( 872 men, 1197 women) visited outpatient clinics of the participating hospitals for an annual health checkup. They had no history of ischemic or hemorrhagic stroke or other cerebral diseases; of coronary heart disease, peripheral arterial occlusive disease, or other atherosclerotic diseases; or of other thrombotic, embolic, or hemorrhagic disorders.

Subjects with ACI and controls either had or did not have conventional risk factors for atherosclerosis, including hypertension (systolic blood pressure of $\geq 140 \mathrm{mmHg}$ or diastolic blood pressure of $\geq 90 \mathrm{mmHg}$, or both), hypercholesterolemia (serum total cholesterol of $\geq 5.72 \mathrm{mmol} / \mathrm{l}$ ), diabetes mellitus (fasting blood glucose of $\geq 6.93 \mathrm{mmol} / \mathrm{l}$ or hemoglobin $\mathrm{A}_{1 \mathrm{c}}$ of $\geq 6.5 \%$, or both), obesity [body mass index (BMI) of $\geq 25 \mathrm{~kg}$ / $\mathrm{m}^{2}$ ], and cigarette smoking ( $\geq 10$ cigarettes daily). The study protocol complied with the Declaration of Helsinki and was approved by the Committees on the Ethics of Human Research of Mie University School of Medicine, Hirosaki University School of Medicine, Gifu International Institute of Biotechnology, and participating hospitals. Written informed consent was obtained from each participant.

Selection of polymorphisms. With the use of public databases, we selected 152 candidate genes that might be associated with ACI on the basis of a comprehensive overview of vascular biology; platelet function; leukocyte, lymphocyte, and monocyte-macrophage biology; coagulation and fibrinolysis cascades; neurological factors; as well as lipid, glucose, and homocysteine metabolism and other metabolic factors. We further selected 202 polymorphisms of these genes, most located in the promoter region, exons, or splice donor or acceptor sites of introns, that might be expected to result in changes in the function or expression of the encoded protein (15).

Genotyping of polymorphisms. Venous blood $(7 \mathrm{ml})$ was collected into tubes containing $50 \mathrm{mmol} / \mathrm{l}$ EDTA (disodium salt), and genomic DNA was isolated with a kit (Genomix; Talent, Trieste, Italy). Genotypes of the 202 polymorphisms were determined (G\&G Science, Fukushima, Japan) by a method that combines the polymerase chain reaction and sequence-specific oligonucleotide probes with the use of suspension array technology (Luminex 100; Luminex, Austin, TX). Detailed methodology for genotyping was described previously (16).

Statistical analysis. Clinical data were compared between subjects with ACI and controls by the unpaired Student's t-test. Qualitative data were compared by the Chi-square test. Allele frequencies were estimated by the gene counting method, and the Chi-square test was used to identify departure from Hardy- 
Table II. Multivariable logistic regression analysis of polymorphisms related to ACI in women.

\begin{tabular}{|c|c|c|c|c|c|c|c|c|c|}
\hline \multirow[b]{2}{*}{ Gene } & \multirow[b]{2}{*}{ Polymorphism } & \multicolumn{2}{|r|}{ Dominant } & \multicolumn{2}{|r|}{ Recessive } & \multicolumn{2}{|c|}{ Additive 1} & \multicolumn{2}{|c|}{ Additive 2} \\
\hline & & $\mathrm{P}$ & OR $(95 \% \mathrm{CI})$ & $\mathrm{P}$ & OR $(95 \% \mathrm{CI})$ & $\mathrm{P}$ & OR $(95 \% \mathrm{CI})$ & $\mathrm{P}$ & OR $(95 \% \mathrm{CI})$ \\
\hline$L I P G$ & $584 \mathrm{C} \rightarrow \mathrm{T}(\mathrm{Thr} 111 \mathrm{Ile})$ & 0.0005 & $1.66(1.25-2.21)$ & 0.1356 & & 0.0012 & $1.63(1.21-2.19)$ & 0.0335 & $1.93(1.03-3.50)$ \\
\hline CCL11 & $\mathrm{G} \rightarrow \mathrm{A}(\mathrm{Ala23Thr})$ & 0.0008 & $0.53(0.36-0.76)$ & 0.2374 & & 0.0016 & $0.54(0.37-0.78)$ & 0.1944 & \\
\hline EDN1 & $5665 \mathrm{G} \rightarrow \mathrm{T}($ Lys198Asn $)$ & 0.0003 & $1.69(1.27-2.25)$ & 0.1755 & & 0.0008 & $1.67(1.24-2.25)$ & 0.0288 & $1.81(1.05-3.04)$ \\
\hline AKAP10 & $\mathrm{A} \rightarrow \mathrm{G}(\mathrm{Ile} 646 \mathrm{Val})$ & 0.0080 & $0.66(0.49-0.90)$ & 0.0537 & & 0.0276 & $0.70(0.51-0.96)$ & 0.0333 & $0.32(0.09-0.82)$ \\
\hline UTS2 & $\mathrm{G} \rightarrow \mathrm{A}($ Ser89Asn $)$ & 0.0175 & $1.42(1.06-1.89)$ & 0.2085 & & 0.0058 & $1.15(1.13-2.03)$ & 0.3707 & \\
\hline IL6 & $-572 \mathrm{G} \rightarrow \mathrm{C}$ & 0.2590 & & 0.0033 & $1.56(1.16-2.10)$ & 0.7415 & & 0.1198 & \\
\hline PTGDS & $4111 \mathrm{~A} \rightarrow \mathrm{C}$ & 0.4035 & & 0.0164 & $0.70(0.52-0.94)$ & 0.1808 & & 0.5660 & \\
\hline ANXA5 & $-1 \mathrm{C} \rightarrow \mathrm{T}$ & 0.1830 & & 0.7426 & & 0.3885 & & 0.7417 & \\
\hline KCNJ11 & $\mathrm{A} \rightarrow \mathrm{G}$ (Glu23Lys) & 0.0058 & $1.93(1.23-3.16)$ & 0.0932 & & 0.0154 & $1.83(0.51-0.96)$ & 0.0040 & $2.08(1.28-3.48)$ \\
\hline
\end{tabular}

OR, odds ratio; CI, confidence interval. Multivariable logistic regression analysis was performed with adjustment for age, BMI, and the prevalence of smoking, hypertension, hypercholesterolemia, and diabetes mellitus. P values of $<0.005$ are shown in bold.

Weinberg equilibrium. In the initial screen, the genotype distribution of each autosomal polymorphism was compared by the Chi-square test $(3 \times 2)$ between subjects with $\mathrm{ACI}$ and controls. For gene polymorphisms located on the X chromosome, allele frequencies were compared by the Chi-square test $(2 \times 2)$. Polymorphisms related $(\mathrm{P}<0.05)$ to ACI were further examined by multivariable logistic regression analysis with adjustment for covariates (with the exception of that used for stratification of subjects), with ACI as a dependent variable and independent variables including age, sex (0: woman; 1 : man), BMI, smoking status (0: nonsmoker; 1: smoker), metabolic variables ( 0 : no history of hypertension, diabetes mellitus, or hypercholesterolemia; 1: positive history), and genotype of each polymorphism. Each genotype was assessed according to dominant, recessive, and additive (additive 1 and 2) genetic models, and the $\mathrm{P}$ value, odds ratio, and 95\% confidence interval were calculated. Additive genetic models comprised two groups: heterozygotes versus wild-type homozygotes for the additive 1 model, and variant homozygotes versus wild-type homozygotes for the additive 2 model. We also performed a stepwise forward selection procedure to examine the effects of genotypes as well as of other covariates on ACI. Given the multiple comparisons of genotypes with ACI, we adopted the criterion of $\mathrm{P}<0.005$ for significant association. For other clinical background data, a $\mathrm{P}$ value of $<0.05$ was considered statistically significant. Statistical significance was examined by two-sided tests, and statistical analyses were performed with JMP software version 5.1 (SAS Institute, Cary, NC).

\section{Results}

Association of polymorphisms with ACI in women or men. Characteristics of female and male subjects are shown in Table I. Among women, age and the prevalence of hypertension, hypercholesterolemia, and diabetes mellitus were greater in subjects with ACI than in controls. Among men, age and the prevalence of hypertension, hypercholesterolemia, and diabetes mellitus were greater, whereas the prevalence of smoking was lower, in subjects with ACI than in controls. The Chi-square test revealed that nine and 16 polymorphisms were related $(\mathrm{P}<0.05)$ to $\mathrm{ACI}$ in women and men, respectively (Supplementary Table I). These polymorphisms were further analyzed for their possible association with ACI. Multivariable logistic regression analysis with adjustment for age, BMI, and the prevalence of smoking, hypertension, hypercholesterolemia, and diabetes mellitus revealed that the $584 \mathrm{C} \rightarrow \mathrm{T}$ polymorphism of LIPG (dominant and additive 1 models), the $\mathrm{G} \rightarrow \mathrm{A}$ polymorphism of $\mathrm{CCL} 11$ (dominant and additive 1 models), the $5665 \mathrm{G} \rightarrow \mathrm{T}$ polymorphism of EDN1 (dominant and additive 1 models), the $-572 \mathrm{G} \rightarrow \mathrm{C}$ polymorphism of $I L 6$ (recessive model), and the $\mathrm{A} \rightarrow \mathrm{G}$ polymorphism of KCNJ11 (additive 2 model) were significantly $(\mathrm{P}<0.005)$ associated with ACI for woman (Table II). For men, the $677 \mathrm{C} \rightarrow \mathrm{T}$ polymorphism of MTHFR (recessive and additive 2 models), the $1323 \mathrm{C} \rightarrow \mathrm{T}$ polymorphism of ITGB2 (dominant model), the $\mathrm{T} \rightarrow \mathrm{G}$ polymorphism of THBS2 (additive 1 model), the 3932T $\rightarrow \mathrm{C}$ polymorphism of $A P O E$ (dominant and additive 1 models), and the $-231 \mathrm{~A} \rightarrow \mathrm{G}$ polymorphism of EDNRA (recessive model) were significantly associated with ACI (Table III). We also performed a stepwise forward selection procedure to examine the effects of genotypes for the genes related to ACI as well as of age, BMI, smoking, hypertension, hypercholesterolemia, and diabetes mellitus on the prevalence of ACI (Table IV). Each genotype was examined according to a dominant or recessive model on the basis of statistical significance in the multivariable logistic regression analysis. In descending order of statistical significance, hypertension, age, LIPG genotype (dominant model), EDN1 genotype (dominant model), CCL11 genotype (dominant model), and diabetes mellitus significantly and independently $(\mathrm{P}<0.005)$ affected the prevalence of ACI in women. In men, diabetes mellitus, age, hypertension, MTHFR genotype (recessive model), ITGB2 genotype (dominant model), APOE genotype (dominant model), and EDNRA genotype (recessive model) significantly and independently affected the prevalence of ACI. 
Table III. Multivariable logistic regression analysis of polymorphisms related to ACI in men.

\begin{tabular}{|c|c|c|c|c|c|c|c|c|c|}
\hline \multirow[b]{2}{*}{ Gene } & \multirow[b]{2}{*}{ Polymorphism } & \multicolumn{2}{|r|}{ Dominant } & \multicolumn{2}{|r|}{ Recessive } & \multicolumn{2}{|c|}{ Additive 1} & \multicolumn{2}{|r|}{ Additive 2} \\
\hline & & $\mathrm{P}$ & OR $(95 \% \mathrm{CI})$ & $\mathrm{P}$ & OR $(95 \% \mathrm{CI})$ & $\mathrm{P}$ & OR $(95 \% \mathrm{CI})$ & $\mathrm{P}$ & OR $(95 \%$ CI $)$ \\
\hline MTHFR & $677 \mathrm{C} \rightarrow \mathrm{T}($ Ala222Val $)$ & 0.5927 & & 0.0002 & $1.85(1.33-2.55)$ & 0.4220 & & 0.0030 & $1.73(1.20-2.48)$ \\
\hline ITGB2 & $1323 \mathrm{C} \rightarrow \mathrm{T}$ & 0.0016 & $1.51(1.17-1.95)$ & 0.0882 & & 0.0051 & $1.46(1.12-1.91)$ & 0.0218 & $1.83(1.08-3.06)$ \\
\hline THBS2 & $\mathrm{T} \rightarrow \mathrm{G}\left(3^{\prime} \mathrm{UTR}\right)$ & 0.0120 & $1.52(1.09-2.09)$ & 0.4171 & & 0.0049 & $1.61(1.15-2.25)$ & 0.5019 & \\
\hline ALOX5 & G $\rightarrow$ A (Glu254Lys) & & & 0.0512 & & 0.0512 & & & \\
\hline IPF1 & $-108 / 3 \mathrm{G} \rightarrow 4 \mathrm{G}$ & 0.0115 & $0.69(0.51-0.92)$ & 0.6660 & & 0.0114 & $0.67(0.49-0.91)$ & 0.0699 & \\
\hline$A P O E$ & 3932T $\rightarrow \mathrm{C}($ Cys112Arg $)$ & 0.0021 & $1.62(1.19-2.20)$ & 0.6337 & & 0.0024 & $1.62(1.19-2.22)$ & 0.5415 & \\
\hline$A P O E$ & $-219 \mathrm{G} \rightarrow \mathrm{T}$ & 0.0121 & $2.02(1.19-3.60)$ & 0.2617 & & 0.0203 & $1.96(1.13-3.56)$ & 0.0112 & $2.07(1.20-3.73)$ \\
\hline KCNJ11 & $\mathrm{A} \rightarrow \mathrm{G}$ (Glu23Lys) & 0.0480 & $0.70(0.50-1.00)$ & 0.0251 & $0.74(0.57-0.96)$ & 0.2051 & & 0.0134 & $0.62(0.42-0.91)$ \\
\hline$A C D C$ & $-11,377 \mathrm{C} \rightarrow \mathrm{G}$ & 0.0286 & $1.33(1.03-1.72)$ & 0.0453 & $1.66(1.00-2.73)$ & 0.0950 & & 0.0211 & $1.82(1.09-3.03)$ \\
\hline APOA1 & $84 \mathrm{~T} \rightarrow \mathrm{C}$ & 0.0505 & & 0.0101 & $1.98(1.17-3.32)$ & 0.2100 & & 0.0057 & $2.11(1.24-3.59)$ \\
\hline $\mathrm{APOH}$ & $341 \mathrm{G} \rightarrow \mathrm{A}($ Ser88Asn $)$ & & & 0.1745 & & 0.1745 & & & \\
\hline SELE & $561 \mathrm{~A} \rightarrow \mathrm{C}($ Ser128Arg $)$ & 0.0236 & $1.73(1.07-2.91)$ & 0.1020 & & 0.0573 & & 0.0964 & \\
\hline TNFSF4 & $\mathrm{A} \rightarrow \mathrm{G}$ & 0.4555 & & 0.0053 & $3.94(1.51-10.67)$ & 0.9612 & & 0.0053 & $3.94(1.51-10.71)$ \\
\hline$F 7$ & $11,496 \mathrm{G} \rightarrow \mathrm{A}(\mathrm{Arg} 353 \mathrm{Gln})$ & 0.3265 & & 0.0898 & & 0.1606 & & 0.0985 & \\
\hline$U C P 3$ & $-55 \mathrm{C} \rightarrow \mathrm{T}$ & 0.5552 & & 0.1807 & & 0.2658 & & 0.3490 & \\
\hline EDNRA & $-231 \mathrm{~A} \rightarrow \mathrm{G}$ & 0.2303 & & 0.0027 & $0.65(0.48-0.86)$ & 0.8513 & & 0.0146 & $0.63(0.44-0.91)$ \\
\hline
\end{tabular}

OR, odds ratio; CI, confidence interval. Multivariable logistic regression analysis was performed with adjustment for age, BMI, and the prevalence of smoking, hypertension, hypercholesterolemia, and diabetes mellitus. P values of $<0.005$ are shown in bold.

Table IV. Effects of genotypes and other characteristics on ACI for women or men as determined by a stepwise forward selection procedure.

\begin{tabular}{|c|c|c|c|c|c|}
\hline \multicolumn{3}{|c|}{ Women } & \multicolumn{3}{|l|}{ Men } \\
\hline Variable & $\mathrm{P}$ & $\mathrm{R}^{2}$ & Variable & $\mathrm{P}$ & $\mathrm{R}^{2}$ \\
\hline Hypertension & $<0.0001$ & 0.0876 & Diabetes mellitus & $<0.0001$ & 0.0337 \\
\hline Age & $<0.0001$ & 0.0158 & Age & $<0.0001$ & 0.0210 \\
\hline$L I P G(C C$ versus $T T+C T)$ & 0.0002 & 0.0102 & Hypertension & $<0.0001$ & 0.0133 \\
\hline$E D N 1(G G$ versus $T T+G T)$ & 0.0002 & 0.0099 & $M T H F R(C C+C T$ versus $T T)$ & 0.0003 & 0.0087 \\
\hline$C C L 11(G G$ versus $A A+G A)$ & 0.0003 & 0.0094 & $I T G B 2(C C$ versus $T T+C T)$ & 0.0019 & 0.0064 \\
\hline Diabetes mellitus & 0.0014 & 0.0074 & $A P O E(T T$ versus $C C+T C)$ & 0.0021 & 0.0062 \\
\hline$A K A P 10(A A$ versus $G G+A G)$ & 0.0060 & 0.0055 & $E D N R A(A A+A G$ versus $G G)$ & 0.0045 & 0.0053 \\
\hline$U T S 2(G G$ versus $A A+G A)$ & 0.0062 & 0.0054 & Smoking & 0.0060 & 0.0050 \\
\hline$K C N J 11(A A$ versus $G G+A G)$ & 0.0068 & 0.0053 & $A P O A l(T T+T C$ versus $C C)$ & 0.0078 & 0.0047 \\
\hline$I L 6(G G+G C$ versus $C C)$ & 0.0070 & 0.0053 & $T N F S F 4(A A+A G$ versus $G G)$ & 0.0083 & 0.0046 \\
\hline \multirow[t]{6}{*}{$P T G D S(A A+A C$ versus $C C)$} & 0.0101 & 0.0048 & $K C N J 11(A A+A G$ versus $G G)$ & 0.0165 & 0.0038 \\
\hline & & & $T H B S 2(T T$ versus $G G+T G)$ & 0.0173 & 0.0037 \\
\hline & & & $A P O E(G G$ versus $T T+G T)$ & 0.0233 & 0.0034 \\
\hline & & & $I P F 1(3 G 3 G$ versus $4 G 4 G+3 G 4 G)$ & 0.0296 & 0.0031 \\
\hline & & & BMI & 0.0361 & 0.0029 \\
\hline & & & $A C D C(C C$ versus $G G+C G)$ & 0.0449 & 0.0027 \\
\hline
\end{tabular}

$\mathrm{R}^{2}$, contribution rate.

Association of polymorphisms with ACI in the absence or presence of hypertension. Characteristics of individuals with or without hypertension are shown in Table V. Among normotensive individuals, age and the prevalence of diabetes mellitus 
Table V. Characteristics of subjects with ACI and controls according to the absence or presence of hypertension.

\begin{tabular}{|c|c|c|c|c|c|c|}
\hline \multirow[b]{2}{*}{ Characteristic } & \multicolumn{3}{|c|}{ Hypertension (-) } & \multicolumn{3}{|c|}{ Hypertension (+) } \\
\hline & ACI & Controls & $\mathrm{P}$ & $\mathrm{ACI}$ & Controls & $\mathrm{P}$ \\
\hline No. of subjects & 195 & 1245 & & 441 & 824 & \\
\hline Age (years) & $66.8 \pm 12.9$ & $60.1 \pm 12.2$ & $<0.0001$ & $67.4 \pm 10.3$ & $65.6 \pm 11.0$ & 0.0040 \\
\hline BMI $\left(\mathrm{kg} / \mathrm{m}^{2}\right)$ & $23.3 \pm 2.5$ & $23.3 \pm 2.8$ & 0.9680 & $23.3 \pm 0.2$ & $23.6 \pm 0.1$ & 0.1480 \\
\hline Smoking (\%) & 13.3 & 15.9 & 0.3487 & 14.3 & 14.9 & 0.7584 \\
\hline Hypercholesterolemia (\%) & 14.9 & 22.5 & 0.0124 & 43.1 & 37.7 & 0.0647 \\
\hline Diabetes mellitus (\%) & 20.0 & 10.8 & 0.0006 & 47.4 & 30.3 & $<0.0001$ \\
\hline
\end{tabular}

Data for age and BMI are means \pm SD.

Table VI. Multivariable logistic regression analysis of polymorphisms related to ACI in hypertensive individuals.

\begin{tabular}{|c|c|c|c|c|c|c|c|c|c|}
\hline \multirow[b]{2}{*}{ Gene } & \multirow[b]{2}{*}{ Polymorphism } & \multicolumn{2}{|r|}{ Dominant } & \multicolumn{2}{|r|}{ Recessive } & \multicolumn{2}{|c|}{ Additive 1} & \multicolumn{2}{|c|}{ Additive 2} \\
\hline & & $\mathrm{P}$ & OR $(95 \% \mathrm{CI})$ & $\mathrm{P}$ & OR $(95 \% \mathrm{CI})$ & $\mathrm{P}$ & OR $(95 \% \mathrm{CI})$ & $\mathrm{P}$ & OR $(95 \% \mathrm{CI})$ \\
\hline IL6 & $-572 \mathrm{G} \rightarrow \mathrm{C}$ & 0.4823 & & 0.0021 & $1.47(1.14-1.87)$ & 0.8442 & & 0.2282 & \\
\hline SLC26A8 & $\mathrm{A} \rightarrow \mathrm{G}(\mathrm{Ile} 639 \mathrm{Val})$ & 0.0270 & $1.31(1.03-1.66)$ & 0.8149 & & 0.0167 & $1.35(1.06-1.74)$ & 0.7254 & \\
\hline GP6 & $13,254 \mathrm{~T} \rightarrow \mathrm{C}$ (Ser219Pro) & 0.0231 & $1.90(1.09-3.31)$ & & & 0.0231 & $1.90(1.09-3.32)$ & & \\
\hline THBS2 & $\mathrm{T} \rightarrow \mathrm{G}\left(3^{\prime} \mathrm{UTR}\right)$ & 0.0262 & $1.41(1.04-1.89)$ & 0.2138 & & 0.0476 & $1.37(1.00-1.86)$ & 0.1785 & \\
\hline TNFSF4 & $A \rightarrow G$ & 0.2489 & & 0.0224 & $3.28(1.22-9.75)$ & 0.5264 & & 0.0205 & $3.35(1.24-9.96)$ \\
\hline PCSK9 & $23,968 \mathrm{~A} \rightarrow \mathrm{G}$ (Glu670Gly) & 0.0172 & $1.67(1.09-2.54)$ & 0.5640 & & 0.0106 & $1.75(1.14-2.69)$ & 0.5925 & \\
\hline$I P F 1$ & $-108 / 3 \mathrm{G} \rightarrow 4 \mathrm{G}$ & 0.0217 & $0.72(0.55-0.95)$ & 0.5605 & & 0.0263 & $0.72(0.53-0.96)$ & 0.0700 & \\
\hline CCR5 & $59,029 \mathrm{G} \rightarrow \mathrm{A}$ & 0.5091 & & 0.0329 & $1.32(1.02-1.71)$ & 0.1409 & & 0.4913 & \\
\hline SELE & $561 \mathrm{~A} \rightarrow \mathrm{C}(\mathrm{Ser} 128 \mathrm{Arg})$ & 0.4518 & & 0.7091 & & 0.7499 & & 0.7089 & \\
\hline$H N F 4 A$ & $\mathrm{~A} \rightarrow \mathrm{G}$ & 0.9618 & & 0.0226 & $1.33(1.04-1.69)$ & 0.4980 & & 0.6268 & \\
\hline
\end{tabular}

OR, odds ratio; CI, confidence interval. Multivariable logistic regression analysis was performed with adjustment for age, sex, BMI, and the prevalence of smoking, hypercholesterolemia, and diabetes mellitus. P values of $<0.005$ are shown in bold.

Table VII. Effects of genotypes and other characteristics on ACI in the absence or presence of hypertension as determined by a stepwise forward selection procedure.

\begin{tabular}{|c|c|c|c|c|c|}
\hline \multicolumn{3}{|c|}{ Hypertension (-) } & \multicolumn{3}{|c|}{ Hypertension (+) } \\
\hline Variable & $\mathrm{P}$ & $\mathrm{R}^{2}$ & Variable & $\mathrm{P}$ & $\mathrm{R}^{2}$ \\
\hline Age & $<0.0001$ & 0.0432 & Diabetes mellitus & $<0.0001$ & 0.0215 \\
\hline Sex & $<0.0001$ & 0.0383 & $I L 6(G G+G C$ versus $C C)$ & 0.0016 & 0.0061 \\
\hline Smoking & 0.0032 & 0.0076 & $S L C 26 A 8(A A$ versus $G G+A G)$ & 0.0081 & 0.0043 \\
\hline$S A H(A A$ versus $G G+A G)$ & 0.0082 & 0.0061 & Age & 0.0112 & 0.0039 \\
\hline Diabetes mellitus & 0.0123 & 0.0055 & $H N F 4 A(A A+A G$ versus $G G)$ & 0.0120 & 0.0039 \\
\hline$M T H F R(C C+C T$ versus $T T)$ & 0.0131 & 0.0054 & $T N F S F 4(A A+A G$ versus $G G)$ & 0.0133 & 0.0038 \\
\hline$A G T R I(A A+A C$ versus $C C)$ & 0.0166 & 0.0050 & Sex & 0.0168 & 0.0035 \\
\hline & & & $T H B S 2(T T$ versus $G G+T G)$ & 0.0200 & 0.0033 \\
\hline & & & $G P 6(T T$ versus $C C+T C)$ & 0.0201 & 0.0033 \\
\hline & & & $I P F 1(3 G 3 G$ versus $4 G 4 G+3 G 4 G)$ & 0.0264 & 0.0030 \\
\hline & & & $P C S K 9(A A$ versus $G G+A G)$ & 0.0340 & 0.0028 \\
\hline & & & $C C R 5(G G+G A$ versus $A A)$ & 0.0493 & 0.0024 \\
\hline
\end{tabular}

$\mathrm{R}^{2}$, contribution rate. 
Table VIII. Characteristics of subjects with ACI and controls according to the absence or presence of hypercholesterolemia.

\begin{tabular}{|c|c|c|c|c|c|c|}
\hline \multirow[b]{2}{*}{ Characteristic } & \multicolumn{3}{|c|}{ Hypercholesterolemia (-) } & \multicolumn{3}{|c|}{ Hypercholesterolemia (+) } \\
\hline & $\mathrm{ACI}$ & Controls & $\mathrm{P}$ & $\mathrm{ACI}$ & Controls & $\mathrm{P}$ \\
\hline No. of subjects & 417 & 1478 & & 219 & 591 & \\
\hline Age (years) & $68.1 \pm 11.6$ & $62.0 \pm 12.3$ & $<0.0001$ & $65.6 \pm 10.1$ & $62.6 \pm 11.3$ & $<0.0001$ \\
\hline BMI $\left(\mathrm{kg} / \mathrm{m}^{2}\right)$ & $23.2 \pm 2.9$ & $23.3 \pm 3.0$ & 0.4270 & $23.6 \pm 3.5$ & $23.7 \pm 3.2$ & 0.5510 \\
\hline Smoking (\%) & 14.6 & 16.6 & 0.3191 & 12.8 & 12.7 & 0.9712 \\
\hline Hypertension (\%) & 60.2 & 34.7 & $<0.0001$ & 86.8 & 52.6 & $<0.0001$ \\
\hline Diabetes mellitus (\%) & 29.7 & 15.0 & $<0.0001$ & 56.6 & 27.8 & $<0.0001$ \\
\hline
\end{tabular}

Data for age and BMI are means \pm SD.

Table IX. Multivariable logistic regression analysis of polymorphisms related to ACI in individuals with hypercholesterolemia.

\begin{tabular}{|c|c|c|c|c|c|c|c|c|c|}
\hline \multirow[b]{2}{*}{ Gene } & \multirow[b]{2}{*}{ Polymorphism } & \multicolumn{2}{|r|}{ Dominant } & \multicolumn{2}{|r|}{ Recessive } & \multicolumn{2}{|r|}{ Additive 1} & \multicolumn{2}{|r|}{ Additive 2} \\
\hline & & $\mathrm{P}$ & OR $(95 \% \mathrm{CI})$ & $\mathrm{P}$ & OR $(95 \% \mathrm{CI})$ & $\mathrm{P}$ & OR $(95 \% \mathrm{CI})$ & $\mathrm{P}$ & OR $(95 \% \mathrm{CI})$ \\
\hline$E N G$ & $\mathrm{C} \rightarrow \mathrm{G}$ (Asp366His) & 0.7932 & & 0.1058 & & 0.7824 & & 0.7954 & \\
\hline$A G T R 2$ & $1675 \mathrm{G} \rightarrow \mathrm{A}$ & 0.0128 & $0.61(0.41-0.90)$ & 0.0388 & $0.69(0.48-0.98)$ & 0.1502 & & 0.0115 & $0.59(0.39-0.88)$ \\
\hline CCL5 & $-28 \mathrm{C} \rightarrow \mathrm{G}$ & 0.0138 & $1.60(1.10-2.32)$ & 0.0285 & $3.22(1.13-9.42)$ & 0.0505 & & 0.0178 & $3.57(1.25-10.51)$ \\
\hline AGTR2 & $3123 \mathrm{C} \rightarrow \mathrm{A}$ & 0.0667 & & 0.0312 & $1.55(1.04-2.31)$ & 0.6131 & & 0.0272 & $1.59(1.05-2.40)$ \\
\hline IL6 & $-572 \mathrm{G} \rightarrow \mathrm{C}$ & 0.9547 & & 0.0051 & $1.66(1.17-2.36)$ & 0.4095 & & 0.5815 & \\
\hline$F A B P 2$ & 2445G $\rightarrow$ A (Ala54Thr) & 0.0321 & $1.46(1.04-2.08)$ & 0.0166 & $1.73(1.10-2.70)$ & 0.1697 & & 0.0063 & $1.98(1.21-3.22)$ \\
\hline HNF4A & $\mathrm{A} \rightarrow \mathrm{G}$ & 0.6508 & & 0.0253 & $1.50(1.05-2.14)$ & 0.1949 & & 0.9900 & \\
\hline$I P F 1$ & $-108 / 3 \mathrm{G} \rightarrow 4 \mathrm{G}$ & 0.0054 & $0.57(0.38-0.85)$ & 0.6411 & & 0.0057 & $0.55(0.36-0.84)$ & 0.0408 & $0.61(0.37-0.98)$ \\
\hline CCL5 & $-403 \mathrm{G} \rightarrow \mathrm{A}$ & 0.0634 & & 0.0011 & $2.15(1.36-3.39)$ & 0.4078 & & 0.0009 & $2.35(1.42-3.89)$ \\
\hline$A D R B 2$ & $46 \mathrm{~A} \rightarrow \mathrm{G}(\mathrm{Arg} 16 \mathrm{Gly})$ & 0.9249 & & 0.0051 & $1.71(1.17-2.50)$ & 0.3534 & & 0.0935 & \\
\hline RECQL2 & $\mathrm{T} \rightarrow \mathrm{C}(\mathrm{Cys} 1367$ Arg $)$ & 0.1417 & & 0.7649 & & 0.0712 & & 0.7670 & \\
\hline$F 7$ & $11,496 \mathrm{G} \rightarrow \mathrm{A}(\mathrm{Arg} 353 \mathrm{Gln})$ & 0.1143 & & 0.7775 & & 0.1967 & & 0.7764 & \\
\hline$A C D C$ & $\mathrm{G} \rightarrow \mathrm{T}$ in intron 2 & 0.0211 & $0.67(0.47-0.94)$ & 0.7404 & & 0.0116 & $0.63(0.44-0.90)$ & 0.7245 & \\
\hline COMT & $\mathrm{G} \rightarrow \mathrm{A}(\mathrm{Val158Met})$ & 0.0012 & $0.57(0.40-0.80)$ & 0.3936 & & 0.0018 & $0.56(0.39-0.80)$ & 0.0724 & \\
\hline
\end{tabular}

OR, odds ratio; CI, confidence interval. Multivariable logistic regression analysis was performed with adjustment for age, sex, BMI, and the prevalence of smoking, hypertension, and diabetes mellitus. P values of $<0.005$ are shown in bold.

were greater, whereas the prevalence of hypercholesterolemia was lower, in subjects with ACI than in controls. Among hypertensive individuals, age and the prevalence of diabetes mellitus were greater in subjects with ACI than in controls. The Chi-square test revealed that five and 10 polymorphisms were related to ACI in normotensive or hypertensive individuals, respectively (Supplementary Table II). Multivariable logistic regression analysis with adjustment for age, sex, BMI, and the prevalence of smoking, hypercholesterolemia, and diabetes mellitus revealed that no polymorphism was associated with ACI among normotensive individuals (Supplementary Table III). Among hypertensive individuals, the $-572 \mathrm{G} \rightarrow \mathrm{C}$ polymorphism of IL6 (recessive model) was significantly associated with ACI (Table VI). A stepwise forward selection procedure revealed that, in descending order of statistical significance, age, sex, and smoking status significantly affected the prevalence of ACI in normotensive individuals (Table VII). For hypertensive individuals, diabetes mellitus and IL6 genotype (recessive model) significantly affected the prevalence of ACI (Table VII).

Association of polymorphisms with ACI in the absence or presence of hypercholesterolemia. Characteristics of subjects with or without hypercholesterolemia are shown in Table VIII. Among individuals with or without hypercholesterolemia, age and the prevalence of hypertension and diabetes mellitus were greater in subjects with ACI than in controls. The Chisquare test revealed that 12 and 14 polymorphisms were related to ACI in the absence or presence of hypercholesterolemia, respectively (Supplementary Table IV). Multivariable 
Table X. Effects of genotypes and other characteristics on ACI in the absence or presence of hypercholesterolemia as determined by a stepwise forward selection procedure.

\begin{tabular}{|c|c|c|c|c|c|}
\hline \multicolumn{3}{|c|}{ Hypercholesterolemia (-) } & \multicolumn{3}{|c|}{ Hypercholesterolemia (+) } \\
\hline Variable & $\mathrm{P}$ & $\mathrm{R}^{2}$ & Variable & $\mathrm{P}$ & $\mathrm{R}^{2}$ \\
\hline Hypertension & $<0.0001$ & 0.0433 & Hypertension & $<0.0001$ & 0.0931 \\
\hline Age & $<0.0001$ & 0.0253 & Diabetes mellitus & $<0.0001$ & 0.0332 \\
\hline Sex & $<0.0001$ & 0.0164 & $A G T R 2(G G$ versus $A A+G A)$ & 0.0016 & 0.0106 \\
\hline$A G T(G G$ versus $A A+G A)$ & 0.0027 & 0.0045 & $C C L 5(G G+G A$ versus $A A)$ & 0.0020 & 0.0101 \\
\hline Diabetes mellitus & 0.0041 & 0.0041 & $A D R B 2(A A+A G$ versus $G G)$ & 0.0036 & 0.0090 \\
\hline$P C S K 9(A A$ versus $G G+A G)$ & 0.0086 & 0.0035 & $C O M T(G G$ versus $A A+G A)$ & 0.0037 & 0.0089 \\
\hline$F 7(G G+G A$ versus $A A)$ & 0.0095 & 0.0034 & $I P F 1(3 G 3 G$ versus $4 G 4 G+3 G 4 G)$ & 0.0051 & 0.0083 \\
\hline$M T H F R(C C+C T$ versus $T T)$ & 0.0109 & 0.0032 & $H N F 4 A(A A+A G$ versus $G G)$ & 0.0077 & 0.0075 \\
\hline$I T G B 2(C C$ versus $T T+C T)$ & 0.0126 & 0.0031 & $I L 6(G G+G C$ versus $C C)$ & 0.0081 & 0.0074 \\
\hline \multirow[t]{3}{*}{ Smoking } & 0.0449 & 0.0020 & Sex & 0.0177 & 0.0060 \\
\hline & & & $A C D C(G G$ versus $T T+G T)$ & 0.0397 & 0.0045 \\
\hline & & & Age & 0.0418 & 0.0044 \\
\hline
\end{tabular}

$\mathrm{R}^{2}$, contribution rate.

Table XI. Characteristics of subjects with ACI and controls according to the absence or presence of diabetes mellitus.

\begin{tabular}{|c|c|c|c|c|c|c|}
\hline \multirow[b]{2}{*}{ Characteristic } & \multicolumn{3}{|c|}{ Diabetes mellitus (-) } & \multicolumn{3}{|c|}{ Diabetes mellitus $(+)$} \\
\hline & ACI & Controls & $\mathrm{P}$ & ACI & Controls & $\mathrm{P}$ \\
\hline No. of subjects & 388 & 1684 & & 248 & 385 & \\
\hline Age (years) & $66.7 \pm 12.1$ & $61.6 \pm 12.1$ & $<0.0001$ & $68.1 \pm 9.3$ & $65.6 \pm 11.2$ & 0.0030 \\
\hline BMI $\left(\mathrm{kg} / \mathrm{m}^{2}\right)$ & $23.3 \pm 2.9$ & $23.4 \pm 2.9$ & 0.3450 & $23.4 \pm 3.4$ & $23.6 \pm 3.6$ & 0.6200 \\
\hline Smoking (\%) & 13.1 & 14.9 & 0.3862 & 15.3 & 18.4 & 0.3075 \\
\hline Hypertension (\%) & 59.8 & 34.1 & $<0.0001$ & 84.3 & 64.9 & $<0.0001$ \\
\hline Hypercholesterolemia (\%) & 24.5 & 25.4 & 0.7207 & 50.0 & 42.6 & 0.0680 \\
\hline
\end{tabular}

Data for age and $\mathrm{BMI}$ are means $\pm \mathrm{SD}$.

logistic regression analysis with adjustment for age, sex, BMI, and the prevalence of smoking, hypertension, and diabetes mellitus revealed that no polymorphism was associated with ACI among individuals without hypercholesterolemia (Supplementary Table V). Among individuals with hypercholesterolemia, the $-403 \mathrm{G} \rightarrow \mathrm{A}$ polymorphism of CCL5 (recessive and additive 2 models) and the $\mathrm{G} \rightarrow \mathrm{A}$ polymorphism of COMT (dominant and additive 1 models) were significantly associated with ACI (Table IX). A stepwise forward selection procedure revealed that hypertension, age, sex, AGT genotype (dominant model), and diabetes mellitus significantly influenced ACI in individuals without hypercholesterolemia, whereas hypertension, diabetes mellitus, AGTR2 genotype (dominant model), CCL5 genotype (recessive model), ADRB2 genotype (recessive model), and COMT genotype (dominant model) significantly affected ACI in individuals with hypercholesterolemia (Table X).
Association of polymorphisms with ACI in the absence or presence of diabetes mellitus. Characteristics of subjects with or without diabetes mellitus are shown in Table XI. Age and the prevalence of hypertension were greater in subjects with ACI than in controls for both nondiabetic and diabetic individuals. The Chi-square test revealed that eight and 14 polymorphisms were related to ACI in nondiabetic and diabetic individuals, respectively (Supplementary Table VI). Multivariable logistic regression analysis with adjustment for age, sex, BMI, and the prevalence of smoking, hypertension, and hypercholesterolemia revealed that no polymorphism was associated with ACI in nondiabetic individuals (Supplementary Table VII). In diabetic individuals, the $3932 \mathrm{~T} \rightarrow \mathrm{C}$ polymorphism of $A P O E$ (dominant and additive 1 models), the $2445 \mathrm{G} \rightarrow \mathrm{A}$ polymorphism of $F A B P 2$ (dominant model), and the $\mathrm{A} \rightarrow \mathrm{G}$ polymorphism of TNFSF4 (additive 2 model) were significantly associated with ACI (Table XII). A stepwise forward selection procedure revealed 
Table XII. Multivariable logistic regression analysis of polymorphisms related to ACI in diabetic individuals.

\begin{tabular}{|c|c|c|c|c|c|c|c|c|c|}
\hline \multirow[b]{2}{*}{ Gene } & \multirow[b]{2}{*}{ Polymorphism } & \multicolumn{2}{|r|}{ Dominant } & \multicolumn{2}{|r|}{ Recessive } & \multicolumn{2}{|r|}{ Additive 1} & \multicolumn{2}{|r|}{ Additive 2} \\
\hline & & $\mathrm{P}$ & OR $(95 \% \mathrm{CI})$ & $\mathrm{P}$ & OR (95\% CI) & $\mathrm{P}$ & OR $(95 \% \mathrm{CI})$ & $\mathrm{P}$ & OR $(95 \% \mathrm{CI})$ \\
\hline$A P O E$ & 3932T $\rightarrow C($ Cys112Arg $)$ & 0.0017 & $1.98(1.29-3.03)$ & 0.9025 & & 0.0014 & 2.03 & 0.8137 & \\
\hline$F A B P 2$ & 2445G $\rightarrow$ A (Ala54Thr) & 0.0038 & $1.65(1.78-2.33)$ & 0.0692 & & 0.0160 & $1.57(1.09-2.26)$ & 0.0107 & $1.94(1.17-3.32)$ \\
\hline TNFSF4 & $\mathrm{A} \rightarrow \mathrm{G}$ & 0.1462 & & 0.0053 & $9.36(2.33-63.04)$ & 0.5350 & & 0.0048 & $9.61(2.38-64.85)$ \\
\hline$A P O E$ & 4070C $\rightarrow \mathrm{T}(\operatorname{Arg} 158 \mathrm{Cys})$ & 0.0812 & & & & 0.0812 & & & \\
\hline$A B C C 8$ & $\mathrm{G} \rightarrow \mathrm{A}(\operatorname{Arg} 1273 \mathrm{Arg})$ & 0.7670 & & 0.7244 & & 0.4686 & & 0.7255 & \\
\hline$G C K$ & $-30 \mathrm{G} \rightarrow \mathrm{A}$ & 0.7928 & & 0.0340 & $0.26(0.06-0.79)$ & 0.3520 & & 0.0422 & $0.27(0.06-0.84)$ \\
\hline COMT & $\mathrm{G} \rightarrow \mathrm{A}($ Val158Met $)$ & 0.0063 & $0.62(0.44-0.87)$ & 0.4109 & & 0.0094 & $0.62(0.35-1.17)$ & 0.1117 & \\
\hline MTHFR & $677 \mathrm{C} \rightarrow \mathrm{T}(\mathrm{Ala} 222 \mathrm{Val})$ & 0.5767 & & 0.0250 & $1.66(1.07-2.58)$ & 0.1624 & & 0.1571 & \\
\hline$A P O H$ & $341 \mathrm{G} \rightarrow \mathrm{A}($ Ser88Asn $)$ & 0.7588 & & & & 0.7588 & & & \\
\hline$E N G$ & $\mathrm{C} \rightarrow \mathrm{G}(\mathrm{Asp} 366 \mathrm{His})$ & 0.7674 & & 0.5544 & & 0.7609 & & 0.7684 & \\
\hline P2RY12 & $744 \mathrm{~T} \rightarrow \mathrm{C}$ & 0.9633 & & 0.0384 & $0.31(0.09-0.86)$ & 0.4371 & & 0.0462 & $0.32(0.09-0.89)$ \\
\hline$G P 1 B A$ & $1018 \mathrm{C} \rightarrow \mathrm{T}(\mathrm{Thr} 145 \mathrm{Met})$ & 0.6972 & & 0.7416 & & 0.9659 & & 0.7415 & \\
\hline GYS1 & $\mathrm{A} \rightarrow \mathrm{G}(\mathrm{Met} 416 \mathrm{Val})$ & 0.0132 & $0.57(0.37-0.88)$ & 0.5491 & & 0.0076 & $0.54(0.34-0.84)$ & 0.6608 & \\
\hline$M M P 2$ & $-1306 \mathrm{C} \rightarrow \mathrm{T}$ & 0.0563 & & 0.7812 & & 0.0321 & $0.59(0.36-0.95)$ & 0.7841 & \\
\hline
\end{tabular}

OR, odds ratio; CI, confidence interval. Multivariable logistic regression analysis was performed with adjustment for age, sex, BMI, and the prevalence of smoking, hypertension, and hypercholesterolemia. P values of $<0.005$ are shown in bold.

Table XIII. Effects of genotypes and other characteristics on ACI in the absence or presence of diabetes mellitus as determined by a stepwise forward selection procedure.

\begin{tabular}{|c|c|c|c|c|c|}
\hline \multicolumn{3}{|c|}{ Diabetes mellitus (-) } & \multicolumn{3}{|c|}{ Diabetes mellitus (+) } \\
\hline Variable & $\mathrm{P}$ & $\mathrm{R}^{2}$ & Variable & $\mathrm{P}$ & $\mathrm{R}^{2}$ \\
\hline Hypertension & $<0.0001$ & 0.0428 & Hypertension & $<0.0001$ & 0.0352 \\
\hline Age & $<0.0001$ & 0.0170 & $A P O E(T T$ versus $C C+T C)$ & 0.0015 & 0.0118 \\
\hline Sex & $<0.0001$ & 0.0140 & Age & 0.0017 & 0.0116 \\
\hline$I L 6(G G+G C$ versus $C C)$ & 0.0049 & 0.0040 & $T N F S F 4(A A+A G$ versus $G G)$ & 0.0019 & 0.0114 \\
\hline$M M P 12(A A$ versus $G G+A G)$ & 0.0083 & 0.0035 & $G C K(G G+G A$ versus $A A)$ & 0.0038 & 0.0099 \\
\hline$C O L 3 A 1(G G+G A$ versus $A A)$ & 0.0113 & 0.0032 & Sex & 0.0045 & 0.0095 \\
\hline$S A H(A A$ versus $G G+A G)$ & 0.0139 & 0.0030 & $F A B P 2(G G$ versus $A A+G A)$ & 0.0092 & 0.0080 \\
\hline$A B C A l(A A+A G$ versus $G G)$ & 0.0237 & 0.0026 & $C O M T(G G$ versus $A A+G A)$ & 0.0115 & 0.0075 \\
\hline \multirow[t]{4}{*}{ Smoking } & 0.0290 & 0.0024 & $M T H F R(C C+C T$ versus $T T)$ & 0.0179 & 0.0066 \\
\hline & & & $P 2 R Y 12(T T+T C$ versus $C C)$ & 0.0250 & 0.0059 \\
\hline & & & Hypercholesterolemia & 0.0326 & 0.0054 \\
\hline & & & $G Y S 1(A A$ versus $G G+A G)$ & 0.0420 & 0.0049 \\
\hline
\end{tabular}

$\mathrm{R}^{2}$, contribution rate.

that hypertension, age, sex, and IL6 genotype (recessive model) significantly affected ACI in nondiabetic individuals (Table XIII). Hypertension, APOE genotype (dominant model), age, TNFSF4 genotype (recessive model), GCK genotype (recessive model), and sex significantly influenced ACI in diabetic individuals (Table XIII).

The polymorphisms significantly $(\mathrm{P}<0.005)$ associated with ACI by both multivariable logistic regression analysis and the stepwise forward selection procedure in women or men, or in individuals with or without hypertension, diabetes mellitus, or hypercholesterolemia, are summarized in Table XIV.

\section{Discussion}

We have examined the relation of 202 polymorphisms to ACI in women or men separately as well as in individuals with or 
Table XIV. Summary of polymorphisms significantly $(\mathrm{P}<0.005)$ associated with ACI as determined by multivariable logistic regression analysis and a stepwise forward selection procedure.

\begin{tabular}{|c|c|c|c|c|}
\hline Sex or risk factor & Gene & Polymorphism & Risk allele & Function of encoded protein \\
\hline \multirow[t]{3}{*}{ Women } & $L I P G$ & $584 \mathrm{C} \rightarrow \mathrm{T}(\mathrm{Thr} 111 \mathrm{Ile})$ & $C$ & $\begin{array}{l}\text { Phospholipase involved in HDL and apolipoprotein A-1 } \\
\text { metabolism }\end{array}$ \\
\hline & EDN1 & $5665 \mathrm{G} \rightarrow \mathrm{T}($ Lys198Asn $)$ & $G$ & Vasoconstrictor produced by vascular endothelial cells \\
\hline & CCL11 & $\mathrm{G} \rightarrow \mathrm{A}(\mathrm{Ala23Thr})$ & $G$ & $\begin{array}{l}\text { Chemokine involved in allergic inflammation and } \\
\text { angiogenesis }\end{array}$ \\
\hline \multirow[t]{4}{*}{ Men } & MTHFR & $677 \mathrm{C} \rightarrow \mathrm{T}($ Ala222Val $)$ & $T$ & Enzyme involved in methylation of homocysteine \\
\hline & $I T G B 2$ & $1323 \mathrm{C} \rightarrow \mathrm{T}$ & $C$ & $\begin{array}{l}\text { Integrin that participates in leukocyte adhesion and } \\
\text { cellular signaling }\end{array}$ \\
\hline & $A P O E$ & 3932T $\rightarrow C($ Cys112Arg $)$ & $T$ & $\begin{array}{l}\text { Ligand for the LDL receptor and the LDL receptor-related } \\
\text { protein }\end{array}$ \\
\hline & EDNRA & $-231 \mathrm{~A} \rightarrow \mathrm{G}$ & $G$ & Endothelin 1 receptor that modulates vascular tone \\
\hline \multicolumn{5}{|l|}{ Hypertension (-) } \\
\hline Hypertension (+) & IL6 & $-572 \mathrm{G} \rightarrow \mathrm{C}$ & $C$ & Cytokine involved in acute inflammatory responses \\
\hline \multicolumn{5}{|l|}{ Hypercholesterolemia (-) } \\
\hline \multirow[t]{2}{*}{ Hypercholesterolemia (+) } & CCL5 & $-403 \mathrm{G} \rightarrow \mathrm{A}$ & $A$ & $\begin{array}{l}\text { Chemoattractant for monocytes, } \mathrm{T} \text { helper cells, and } \\
\text { eosinophils }\end{array}$ \\
\hline & COMT & $\mathrm{G} \rightarrow \mathrm{A}($ Val158Met $)$ & $G$ & Enzyme that catalyzes catecholamine transmitters \\
\hline \multicolumn{5}{|l|}{ Diabetes mellitus (-) } \\
\hline \multirow[t]{2}{*}{ Diabetes mellitus (+) } & $A P O E$ & 3932T $\rightarrow C($ Cys112Arg $)$ & $T$ & See above \\
\hline & TNFSF4 & $A \rightarrow G$ & $G$ & $\begin{array}{l}\text { Ligand that mediates adhesion of activated } \mathrm{T} \text { cells to } \\
\text { endothelial cells }\end{array}$ \\
\hline
\end{tabular}

HDL, high density lipoprotein; LDL, low density lipoprotein.

without hypertension, hypercholesterolemia, or diabetes mellitus. Our observations suggest that polymorphisms associated with ACI may differ between men and women as well as among individuals with or without different conventional risk factors for atherosclerosis.

ACI is the most common type of stroke and, in most patients, is caused by atherosclerosis (5). Atherosclerosis results from excessive inflammatory and fibroproliferative responses to various forms of insult to the endothelium and smooth muscle of the artery wall, with the participation of large numbers of growth factors, cytokines, and vasoregulatory molecules (17). We therefore selected 152 candidate genes for ACI on the basis of a comprehensive overview of vascular, platelet, leukocyte, lymphocyte, and monocyte-macrophage biology; coagulation and fibrinolysis cascades; neurological factors; as well as lipid, glucose, and homocysteine metabolism and other metabolic factors. Indeed, the genes found to be associated with ACI may have roles in diverse aspects of the etiology of this condition, including cell adhesion (ITGB2, TNFSF4); vascular inflammation (CCL11, IL6); leukocyte, lymphocyte, and monocyte-macrophage biology (CCL5); vascular constriction (EDN1, EDNRA); and metabolism of lipids ( $L I P G, A P O E)$, homocysteine (MTHFR), and catecholamine transmitters $(C O M T)$. Three of the 11 polymorphisms associated with ACI in the present study $(677 \mathrm{C} \rightarrow \mathrm{T}$ of $M T H F R$, $3932 \mathrm{~T} \rightarrow \mathrm{C}$ of $A P O E$, and $-572 \mathrm{G} \rightarrow \mathrm{C}$ of $I L 6)$ have previously been associated with ischemic stroke $(8,9,15,18,19)$. The remaining eight polymorphisms $(584 \mathrm{C} \rightarrow \mathrm{T}$ of $L I P G, 5665 \mathrm{G} \rightarrow \mathrm{T}$ of $E D N 1, \mathrm{G} \rightarrow \mathrm{A}$ of $C C L 11,1323 \mathrm{C} \rightarrow \mathrm{T}$ of $I T G B 2,-231 \mathrm{~A} \rightarrow \mathrm{G}$ of EDNRA, $-403 \mathrm{G} \rightarrow \mathrm{A}$ of $C C L 5, \mathrm{G} \rightarrow \mathrm{A}$ of $C O M T$, and $\mathrm{A} \rightarrow \mathrm{G}$ of TNFSF4) have not been previously associated with this condition.

Association of polymorphisms with ACI in women versus men. The $584 \mathrm{C} \rightarrow \mathrm{T}$ polymorphism of $L I P G$, the $5665 \mathrm{G} \rightarrow \mathrm{T}$ polymorphism of $E D N 1$, and the $\mathrm{G} \rightarrow \mathrm{A}$ polymorphism of CCL11 were associated with ACI in women, whereas the $677 \mathrm{C} \rightarrow \mathrm{T}$ polymorphism of $M T H F R$, the $1323 \mathrm{C} \rightarrow \mathrm{T}$ polymorphism of ITGB2, the $3932 \mathrm{~T} \rightarrow \mathrm{C}$ polymorphism of $A P O E$, and the $-231 \mathrm{~A} \rightarrow \mathrm{G}$ polymorphism of $E D N R A$ were associated with this condition in men. The mechanisms responsible for the difference in the polymorphisms associated with ACI between men and women remain unclear. Given that, in general, the total risk for atherosclerotic disease, such as coronary heart disease and ACI, in women lags behind that in men by approximately 10 years, the mechanisms underlying the risk for ACI in women may differ from those in men at each age. The sex difference in the association of poly- 
morphisms with ACI might be attributable to the difference in sex hormones such as estrogen between men and women, given that estrogen exerts various favorable effects on vessel wall and vasomotor function, including stimulation of the production of nitric oxide and prostaglandin $\mathrm{I}_{2}$ as well as inhibition of the release of endothelin- 1 by vascular endothelial cells (20). Furthermore, considering that the polymorphisms examined in our study probably represent only a small proportion of those potentially associated with ACI, it remains possible that further investigations will uncover polymorphisms that are associated with ACI in both men and women.

Association of polymorphisms with ACI in the absence or presence of conventional risk factors for atherosclerosis. Given that interactions between gene polymorphisms and conventional risk factors may be important in the etiology of ACI, we examined the effects of polymorphisms on the prevalence of ACI in the absence or presence of hypertension, hypercholesterolemia, or diabetes mellitus. The $-572 \mathrm{G} \rightarrow \mathrm{C}$ polymorphism of IL6 was associated with ACI in hypertensive individuals, whereas no polymorphism was associated with $\mathrm{ACI}$ in normotensive individuals. The $-403 \mathrm{G} \rightarrow \mathrm{A}$ polymorphism of CCL5 and the $\mathrm{G} \rightarrow \mathrm{A}$ polymorphism of COMT were associated with ACI in subjects with hypercholesterolemia, whereas no polymorphism was associated with ACI in subjects without hypercholesterolemia. The $3932 \mathrm{~T} \rightarrow \mathrm{C}$ polymorphism of $A P O E$ and the $\mathrm{A} \rightarrow \mathrm{G}$ polymorphism of TNFSF4 were associated with $\mathrm{ACI}$ in diabetic individuals, whereas no polymorphism was associated with this condition in nondiabetic subjects. These observations suggest that polymorphisms associated with ACI may differ among subjects with different conventional risk factors, although the mechanisms responsible for these differences remain to be elucidated. Given that the effects of single polymorphisms on the development of ACI are likely to be small, the association between a polymorphism and the prevalence of ACI might be influenced by the absence or presence of conventional risk factors for atherosclerosis. Furthermore, considering that hypertension, hypercholesterolemia, and diabetes mellitus probably have genetic components, there might be interactions between genes related to ACI and those related to conventional risk factors.

There were several limitations to the present study. The number of subjects with ACI was relatively small after stratification of these individuals by sex or conventional risk factors. Considering the multiple comparisons of genotypes with ACI, we adopted the criterion of $\mathrm{P}<0.005$ for significant association. However, this approach does not completely exclude the possibility of false positive associations. It is also possible that one or more of the polymorphisms associated with ACI in our study are in linkage disequilibrium with polymorphisms of other nearby genes that are actually responsible for the development of this condition. Furthermore, the functional relevance of the identified polymorphisms to gene transcription or to protein structure or function was not determined in the present study.

Our present observations suggest that polymorphisms associated with ACI may differ between women and men as well as among individuals with different conventional risk factors for atherosclerosis. Stratification of subjects on the basis of sex or conventional risk factors may thus be important in order to achieve the personalized prevention of ACI with the use of genetic information.

\section{Acknowledgments}

This work was supported in part by Grants-in-Aid for Scientific Research on Priority Areas (Applied Genomics) from the Ministry of Education, Culture, Sports, Science, and Technology of Japan (nos. 16012226, 17019030, 18018021) as well as by grants from Gifu Prefectural Science and Technology Promotion Center, Okasan Kato Culture Promotion Foundation, Japan Cardiovascular Research Foundation, Takeda Science Foundation, Daiwa Securities Health Foundation, and Mitsubishi Pharma Research Foundation, all to Y.Y.

\section{References}

1. Warlow C, Sudlow C, Dennis M, Wardlaw J and Sandercock P: Stroke. Lancet 362: 1211-1224, 2003.

2. Thom T, Haase N, Rosamond W, Howard VJ, Rumsfeld J, Manolio T, Zheng ZJ, Flegal K, O'Donnell C, Kittner S, Lloyd-Jones D, Goff DC Jr, Hong Y, Adams R, Friday G, Furie K, Gorelick P, Kissela B, Marler J, Meigs J, Roger V, Sidney S, Sorlie P, Steinberger J, Wasserthiel-Smoller S, Wilson M and Wolf P; American Heart Association Statistics Committee and Stroke Statistics Subcommittee: Heart disease and stroke statistics - 2006 update: a report from the American Heart Association Statistics Committee and Stroke Statistics Subcommittee. Circulation 113: e85-e151, 2006.

3. Goldstein LB, Adams R, Becker K, Furberg CD, Gorelick PB, Hademenos G, Hill M, Howard G, Howard VJ, Jacobs B, Levine SR, Mosca L, Sacco RL, Sherman DG, Wolf PA, and del Zoppo GJ: Primary prevention of ischemic stroke: a statement for healthcare professionals from the Stroke Council of the American Heart Association. Stroke 32: 280-299, 2001.

4. Hassan A and Markus HS: Genetics and ischaemic stroke. Brain 123: 1784-1812, 2000.

5. Humphries SE and Morgan L: Genetic risk factors for stroke and carotid atherosclerosis: insights into pathophysiology from candidate gene approaches. Lancet Neurol 3: 227-235, 2004.

6. Pola R, Flex A, Gaetani E, Flore R, Serricchio M and Pola P: Synergistic effect of -174 G/C polymorphism of the interleukin-6 gene promoter and $469 \mathrm{E} / \mathrm{K}$ polymorphism of the intercellular adhesion molecule-1 gene in Italian patients with history of ischemic stroke. Stroke 34: 881-885, 2003.

7. Flex A, Gaetani E, Papaleo P, Straface G, Proia AS, Pecorini G, Tondi P, Pola P and Pola R: Proinflammatory genetic profiles in subjects with history of ischemic stroke. Stroke 35: 2270-2275, 2004.

8. Morita H, Kurihara H, Tsubaki S, Sugiyama T, Hamada C, Kurihara Y, Shindo T, Oh-hashi Y, Kitamura K and Yazaki Y: Methylenetetrahydrofolate reductase gene polymorphism and ischemic stroke in Japanese. Arterioscler Thromb Vasc Biol 18: 1465-1469, 1998.

9. Kohara K, Fujisawa M, Ando F, Tabara Y, Niino N, Miki T and Shimokata H; NILS-LSA Study: MTHFR gene polymorphism as a risk factor for silent brain infarcts and white matter lesions in the Japanese general population: The NILS-LSA Study. Stroke 34: 1130-1135, 2003.

10. Voetsch B, Benke KS, Damasceno BP, Siqueira LH and Loscalzo J: Paraoxonase $192 \mathrm{Gln} \rightarrow$ Arg polymorphism: an independent risk factor for nonfatal arterial ischemic stroke among young adults. Stroke 33: 1459-1464, 2002.

11. Gretarsdottir S, Thorleifsson G, Reynisdottir ST, Manolescu A, Jonsdottir S, Jonsdottir T, Gudmundsdottir T, Bjarnadottir SM, Einarsson OB, Gudjonsdottir HM, Hawkins M, Gudmundsson G, Gudmundsdottir H, Andrason H, Gudmundsdottir AS, Sigurdardottir M, Chou TT, Nahmias J, Goss S, Sveinbjornsdottir S, Valdimarsson EM, Jakobsson F, Agnarsson U, Gudnason V, Thorgeirsson G, Fingerle J, Gurney M, Gudbjartsson D, Frigge ML, Kong A, Stefansson K and Gulcher JR: The gene encoding phosphodiesterase 4D confers risk of ischemic stroke. Nat Genet 35: 131-138, 2003. 
12. Helgadottir A, Manolescu A, Thorleifsson G, Gretarsdottir S, Jonsdottir H, Thorsteinsdottir U, Samani NJ, Gudmundsson G, Grant SF, Thorgeirsson G, Sveinbjornsdottir S, Valdimarsson EM, Matthiasson SE, Johannsson H, Gudmundsdottir O, Gurney ME, Sainz J, Thorhallsdottir M, Andresdottir M, Frigge ML, Topol EJ, Kong A, Gudnason V, Hakonarson H, Gulcher JR and Stefansson K: The gene encoding 5-lipoxygenase activating protein confers risk of myocardial infarction and stroke. Nat Genet 36: 233-239, 2004.

13. Cipollone F, Toniato E, Martinotti S, Fazia M, Iezzi A, Cuccurullo C, Pini B, Ursi S, Vitullo G, Averna M, Arca M, Montali A, Campagna F, Ucchino S, Spigonardo F, Taddei S, Virdis A, Ciabattoni G, Notarbartolo A, Cuccurullo F and Mezzetti A; Identification of New Elements of Plaque Stability (INES) Study Group: A polymorphism in the cyclooxygenase 2 gene as an inherited protective factor against myocardial infarction and stroke. JAMA 291: 2221-2228, 2004.

14. Special report from the National Institute of Neurological Disorders and Stroke. Classification of cerebrovascular diseases III. Stroke 21: 637-676, 1990.

15. Yamada Y, Metoki N, Yoshida H, Satoh K, Ichihara S, Kato K, Kameyama T, Yokoi K, Matsuo H, Segawa T, Watanabe S and Nozawa Y: Genetic risk for ischemic and hemorrhagic stroke. Arterioscler Thromb Vasc Biol 26: 1920-1925, 2006.
16. Itoh Y, Mizuki N, Shimada T, Azuma F, Itakura M, Kashiwase K, Kikkawa E, Kulski JK, Satake M and Inoko H: High throughput DNA typing of HLA-A, -B, -C and -DRB1 loci by a PCR-SSOPLuminex method in the Japanese population. Immunogenetics 57: 717-729, 2005.

17. Ross R: The pathogenesis of atherosclerosis: a perspective for the 1990s. Nature 362: 801-809, 1993.

18. Kessler C, Spitzer C, Stauske D, Mende S, Stadlmuller J, Walther R and Rettig R: The apolipoprotein E and betafibrinogen G/A-455 gene polymorphisms are associated with ischemic stroke involving large-vessel disease. Arterioscler Thromb Vasc Biol 17: 2880-2884, 1997.

19. Szolnoki Z, Somogyvari F, Kondacs A, Szabo M, Fodor L, Bene $J$ and Melegh B: Evaluation of the modifying effects of unfavourable genotypes on classical clinical risk factors for ischaemic stroke. J Neurol Neurosurg Psychiatry 74: 1615-1620, 2003

20. Koh KK: Effects of estrogen on the vascular wall: vasomotor function and inflammation. Cardiovasc Res 55: 714-726, 2002.

Supplementary Table I. Polymorphisms related to ACI in women or men as determined by the Chi-square test.

\begin{tabular}{|c|c|c|c|c|c|}
\hline \multicolumn{3}{|c|}{ Women } & \multicolumn{3}{|c|}{ Men } \\
\hline Gene & Polymorphism & $\mathrm{P}$ & Gene & Polymorphism & $\mathrm{P}$ \\
\hline$L I P G$ & $584 \mathrm{C} \rightarrow \mathrm{T}(\mathrm{Thr} 111 \mathrm{Ile})$ & 0.0007 & MTHFR & $677 \mathrm{C} \rightarrow \mathrm{T}(\mathrm{Ala} 222 \mathrm{Val})$ & 0.0011 \\
\hline CCL11 & $\mathrm{G} \rightarrow \mathrm{A}(\mathrm{Ala23Thr})$ & 0.0010 & $I T G B 2$ & $1323 \mathrm{C} \rightarrow \mathrm{T}$ & 0.0031 \\
\hline EDN1 & $5665 \mathrm{G} \rightarrow \mathrm{T}($ Lys 198Asn $)$ & 0.0027 & THBS2 & $\mathrm{T} \rightarrow \mathrm{G}\left(3^{\prime} \mathrm{UTR}\right)$ & 0.0050 \\
\hline AKAP10 & $\mathrm{A} \rightarrow \mathrm{G}(\mathrm{Ile} 646 \mathrm{Val})$ & 0.0044 & $A L O X 5$ & G $\rightarrow$ A (Glu254Lys) & 0.0083 \\
\hline UTS2 & $\mathrm{G} \rightarrow \mathrm{A}($ Ser89Asn $)$ & 0.0120 & IPF1 & $-108 / 3 G \rightarrow 4 G$ & 0.0220 \\
\hline IL6 & $-572 \mathrm{G} \rightarrow \mathrm{C}$ & 0.0186 & $A P O E$ & 3932T $\rightarrow \mathrm{C}(\mathrm{Cys} 112 \mathrm{Arg})$ & 0.0284 \\
\hline PTGDS & $4111 \mathrm{~A} \rightarrow \mathrm{C}$ & 0.0195 & $A P O E$ & $-219 \mathrm{G} \rightarrow \mathrm{T}$ & 0.0320 \\
\hline ANXA5 & $-1 \mathrm{C} \rightarrow \mathrm{T}$ & 0.0197 & KCNJ11 & $\mathrm{A} \rightarrow \mathrm{G}$ (Glu23Lys) & 0.0327 \\
\hline KCNJ11 & $\mathrm{A} \rightarrow \mathrm{G}$ (Glu23Lys) & 0.0210 & $A C D C$ & $-11,377 \mathrm{C} \rightarrow \mathrm{G}$ & 0.0327 \\
\hline & & & APOA1 & $84 \mathrm{~T} \rightarrow \mathrm{C}$ & 0.0355 \\
\hline & & & APOH & $341 \mathrm{G} \rightarrow \mathrm{A}($ Ser88Asn $)$ & 0.0355 \\
\hline & & & SELE & $561 \mathrm{~A} \rightarrow \mathrm{C}(\operatorname{Ser} 128 \mathrm{Arg})$ & 0.0358 \\
\hline & & & TNFSF4 & $\mathrm{A} \rightarrow \mathrm{G}$ & 0.0369 \\
\hline & & & $F 7$ & $11,496 \mathrm{G} \rightarrow \mathrm{A}(\mathrm{Arg} 353 \mathrm{Gln})$ & 0.0387 \\
\hline & & & UCP3 & $-55 \mathrm{C} \rightarrow \mathrm{T}$ & 0.0468 \\
\hline & & & EDNRA & $-231 \mathrm{~A} \rightarrow \mathrm{G}$ & 0.0468 \\
\hline
\end{tabular}

Supplementary Table II. Polymorphisms related to ACI in the absence or presence of hypertension as determined by the Chisquare test.

\begin{tabular}{|c|c|c|c|c|c|}
\hline \multicolumn{3}{|c|}{ Hypertension (-) } & \multicolumn{3}{|c|}{ Hypertension (+) } \\
\hline Gene & Polymorphism & $\mathrm{P}$ & Gene & Polymorphism & $\mathrm{P}$ \\
\hline MTHFR & $677 \mathrm{C} \rightarrow \mathrm{T}(\mathrm{Ala} 222 \mathrm{Val})$ & 0.0070 & IL6 & $-572 \mathrm{G} \rightarrow \mathrm{C}$ & 0.0067 \\
\hline NOS3 & $-786 \mathrm{~T} \rightarrow \mathrm{C}$ & 0.0241 & SLC26A8 & $\mathrm{A} \rightarrow \mathrm{G}(\mathrm{Ile} 639 \mathrm{Val})$ & 0.0149 \\
\hline$S A H$ & $A \rightarrow G$ in intron 12 & 0.0418 & GP6 & $13,254 \mathrm{~T} \rightarrow \mathrm{C}($ Ser219Pro $)$ & 0.0268 \\
\hline$U C P 3$ & $-55 \mathrm{C} \rightarrow \mathrm{T}$ & 0.0473 & THBS2 & $\mathrm{T} \rightarrow \mathrm{G}\left(3^{\prime} \mathrm{UTR}\right)$ & 0.0316 \\
\hline AGTRl & $1166 \mathrm{~A} \rightarrow \mathrm{C}$ & 0.0497 & TNFSF4 & $A \rightarrow G$ & 0.0329 \\
\hline & & & PCSK9 & 23,968A $\rightarrow \mathrm{G}$ (Glu670Gly) & 0.0329 \\
\hline & & & $I P F 1$ & $-108 / 3 \mathrm{G} \rightarrow 4 \mathrm{G}$ & 0.0362 \\
\hline & & & CCR5 & $59,029 \mathrm{G} \rightarrow \mathrm{A}$ & 0.0367 \\
\hline & & & SELE & $561 \mathrm{~A} \rightarrow \mathrm{C}(\operatorname{Ser} 128 \mathrm{Arg})$ & 0.0404 \\
\hline & & & $H N F 4 A$ & $\mathrm{~A} \rightarrow \mathrm{G}$ & 0.0465 \\
\hline
\end{tabular}


Supplementary Table III. Multivariable logistic regression analysis of polymorphisms related to ACI in normotensive individuals.

\begin{tabular}{|c|c|c|c|c|c|c|c|c|c|}
\hline \multirow[b]{2}{*}{ Gene } & \multirow[b]{2}{*}{ Polymorphism } & \multicolumn{2}{|r|}{ Dominant } & \multicolumn{2}{|r|}{ Recessive } & \multicolumn{2}{|r|}{ Additive 1} & \multicolumn{2}{|r|}{ Additive 2} \\
\hline & & $\mathrm{P}$ & OR $(95 \% \mathrm{CI})$ & $\mathrm{P}$ & OR $(95 \% \mathrm{CI})$ & $\mathrm{P}$ & OR $(95 \% \mathrm{CI})$ & $\mathrm{P}$ & OR $(95 \% \mathrm{CI})$ \\
\hline MTHFR & $677 \mathrm{C} \rightarrow \mathrm{T}(\mathrm{Ala} 222 \mathrm{Val})$ & 0.9203 & & 0.0073 & $1.69(1.14-2.47)$ & 0.3760 & & 0.0495 & $1.54(1.00-2.38)$ \\
\hline NOS3 & $-786 \mathrm{~T} \rightarrow \mathrm{C}$ & 0.0731 & & 0.7701 & & 0.1461 & & 0.7688 & \\
\hline$S A H$ & $\mathrm{~A} \rightarrow \mathrm{G}$ in intron 12 & 0.0323 & $0.32(0.10-0.81)$ & 0.8734 & & 0.0334 & $0.32(0.10-0.81)$ & 0.8721 & \\
\hline$U C P 3$ & $-55 \mathrm{C} \rightarrow \mathrm{T}$ & 0.4095 & & 0.0964 & & 0.1473 & & 0.2580 & \\
\hline AGTRI & $1166 \mathrm{~A} \rightarrow \mathrm{C}$ & 0.2329 & & 0.0095 & $4.96(1.39-16.53)$ & 0.5319 & & 0.0087 & $5.08(1.42-16.95)$ \\
\hline
\end{tabular}

OR, odds ratio; CI, confidence interval. Multivariable logistic regression analysis was performed with adjustment for age, sex, BMI, and the prevalence of smoking, hypercholesterolemia, and diabetes mellitus.

Supplementary Table IV. Polymorphisms related to ACI in the absence or presence of hypercholesterolemia as determined by the Chi-square test

\begin{tabular}{|c|c|c|c|c|c|}
\hline \multicolumn{3}{|c|}{ Hypercholesterolemia (-) } & \multicolumn{3}{|c|}{ Hypercholesterolemia (+) } \\
\hline Gene & Polymorphism & $\mathrm{P}$ & Gene & Polymorphism & $\mathrm{P}$ \\
\hline$F 7$ & $11,496 \mathrm{G} \rightarrow \mathrm{A}(\mathrm{Arg} 353 \mathrm{Gln})$ & 0.0057 & $E N G$ & $\mathrm{C} \rightarrow \mathrm{G}(\mathrm{Asp} 366 \mathrm{His})$ & 0.0003 \\
\hline$A G T$ & $-6 \mathrm{G} \rightarrow \mathrm{A}$ & 0.0076 & AGTR2 & $1675 \mathrm{G} \rightarrow \mathrm{A}$ & 0.0037 \\
\hline MTHFR & 677C $\rightarrow \mathrm{T}(\mathrm{Ala} 222 \mathrm{Val})$ & 0.0088 & CCL5 & $-28 \mathrm{C} \rightarrow \mathrm{G}$ & 0.0046 \\
\hline ITGB2 & $1323 \mathrm{C} \rightarrow \mathrm{T}$ & 0.0095 & $A G T R 2$ & $3123 \mathrm{C} \rightarrow \mathrm{A}$ & 0.0058 \\
\hline UTS2 & $\mathrm{G} \rightarrow \mathrm{A}($ Ser89Asn $)$ & 0.0128 & IL6 & $-572 \mathrm{G} \rightarrow \mathrm{C}$ & 0.0076 \\
\hline$A N X A 5$ & $-1 \mathrm{C} \rightarrow \mathrm{T}$ & 0.0143 & $F A B P 2$ & 2445G $\rightarrow$ A (Ala54Thr) & 0.0133 \\
\hline SELE & $561 \mathrm{~A} \rightarrow \mathrm{C}(\operatorname{Ser} 128 \mathrm{Arg})$ & 0.0214 & $H N F 4 A$ & $\mathrm{~A} \rightarrow \mathrm{G}$ & 0.0228 \\
\hline$L I P G$ & 584C $\rightarrow \mathrm{T}(\mathrm{Thr} 111 \mathrm{Ile})$ & 0.0252 & $I P F 1$ & $-108 / 3 \mathrm{G} \rightarrow 4 \mathrm{G}$ & 0.0292 \\
\hline$U C P 3$ & $-55 \mathrm{C} \rightarrow \mathrm{T}$ & 0.0374 & CCL5 & $-403 \mathrm{G} \rightarrow \mathrm{A}$ & 0.0300 \\
\hline IL6 & $-572 \mathrm{G} \rightarrow \mathrm{C}$ & 0.0382 & $A D R B 2$ & 46A $\rightarrow \mathrm{G}($ Arg 16Gly) & 0.0375 \\
\hline PAII & $\mathrm{A} \rightarrow \mathrm{G}$ (Tyr243Cys) & 0.0459 & RECQL2 & $\mathrm{T} \rightarrow \mathrm{C}(\mathrm{Cys} 1367$ Arg $)$ & 0.0376 \\
\hline \multirow[t]{3}{*}{ PCSK 9} & 23,968A $\rightarrow \mathrm{G}$ (Glu670Gly) & 0.0467 & F7 & $11,496 \mathrm{G} \rightarrow \mathrm{A}(\mathrm{Arg} 353 \mathrm{Gln})$ & 0.0379 \\
\hline & & & $A C D C$ & $\mathrm{G} \rightarrow \mathrm{T}$ in intron 2 & 0.0425 \\
\hline & & & COMT & $\mathrm{G} \rightarrow \mathrm{A}(\mathrm{Val} 158 \mathrm{Met})$ & 0.0431 \\
\hline
\end{tabular}

Supplementary Table V. Multivariable logistic regression analysis of polymorphisms related to ACI in individuals without hypercholesterolemia.

\begin{tabular}{|c|c|c|c|c|c|c|c|c|c|}
\hline \multirow[b]{2}{*}{ Gene } & \multirow[b]{2}{*}{ Polymorphism } & \multicolumn{2}{|r|}{ Dominant } & \multicolumn{2}{|r|}{ Recessive } & \multicolumn{2}{|r|}{ Additive 1} & \multicolumn{2}{|r|}{ Additive 2} \\
\hline & & $\mathrm{P}$ & OR $(95 \% \mathrm{CI})$ & $\mathrm{P}$ & OR $(95 \% \mathrm{CI})$ & $\mathrm{P}$ & OR $(95 \% \mathrm{CI})$ & $\mathrm{P}$ & OR $(95 \% \mathrm{CI})$ \\
\hline$F 7$ & $11,496 \mathrm{G} \rightarrow \mathrm{A}(\mathrm{Arg} 353 \mathrm{Gln})$ & 0.9484 & & 0.0222 & $7.03(1.50-50.38)$ & 0.6667 & & 0.0229 & $6.97(1.48-49.92)$ \\
\hline$A G T$ & $-6 \mathrm{G} \rightarrow \mathrm{A}$ & 0.0124 & $3.01(1.37-7.95)$ & 0.4913 & & 0.0063 & $3.41(1.52-9.12)$ & 0.0183 & $2.84(1.29-7.53)$ \\
\hline MTHFR & $677 \mathrm{C} \rightarrow \mathrm{T}(\mathrm{Ala} 222 \mathrm{Val})$ & 0.7596 & & 0.0115 & $1.46(1.09-1.96)$ & 0.2160 & & 0.0817 & \\
\hline$I T G B 2$ & $1323 \mathrm{C} \rightarrow \mathrm{T}$ & 0.0146 & $1.33(1.06-1.67)$ & 0.6545 & & 0.0082 & $1.38(1.09-1.74)$ & 0.9249 & \\
\hline UTS2 & $\mathrm{G} \rightarrow \mathrm{A}($ Ser89Asn $)$ & 0.2625 & & 0.0496 & $0.51(0.25-0.96)$ & 0.0903 & & 0.0861 & \\
\hline ANXA5 & $-1 \mathrm{C} \rightarrow \mathrm{T}$ & 0.4968 & & 0.6264 & & 0.7900 & & 0.6261 & \\
\hline SELE & $561 \mathrm{~A} \rightarrow \mathrm{C}(\operatorname{Ser} 128 \mathrm{Arg})$ & 0.1094 & & 0.1456 & & 0.1880 & & 0.1413 & \\
\hline$L I P G$ & $584 \mathrm{C} \rightarrow \mathrm{T}(\mathrm{Thr111 \textrm {Ile }})$ & 0.0414 & $1.27(1.01-1.60)$ & 0.0986 & & 0.0961 & & 0.0501 & \\
\hline$U C P 3$ & $-55 \mathrm{C} \rightarrow \mathrm{T}$ & 0.5128 & & 0.1079 & & 0.2105 & & 0.2496 & \\
\hline IL6 & $-572 \mathrm{G} \rightarrow \mathrm{C}$ & 0.0913 & & 0.0813 & & 0.1941 & & 0.0624 & \\
\hline PAII & $A \rightarrow G($ Tyr243Cys $)$ & 0.7340 & & & & 0.7340 & & & \\
\hline PCSK 9 & $23,968 \mathrm{~A} \rightarrow \mathrm{G}$ (Glu670Gly) & 0.0087 & $1.64(1.13-2.36)$ & 0.8706 & & 0.0064 & $1.69(1.15-2.45)$ & 0.9131 & \\
\hline
\end{tabular}

OR, odds ratio; CI, confidence interval. Multivariable logistic regression analysis was performed with adjustment for age, sex, BMI, and the prevalence of smoking, hypertension, and diabetes mellitus. 
Supplementary Table VI. Polymorphisms related to ACI in the absence or presence of diabetes mellitus as determined by the Chi-square test.

\begin{tabular}{|c|c|c|c|c|c|}
\hline \multicolumn{3}{|c|}{ Diabetes mellitus (-) } & \multicolumn{3}{|c|}{ Diabetes mellitus (+) } \\
\hline Gene & Polymorphism & $\mathrm{P}$ & Gene & Polymorphism & $\mathrm{P}$ \\
\hline$M M P 12$ & $-82 A \rightarrow G$ & 0.0040 & $A P O E$ & 3932T $\rightarrow \mathrm{C}(\mathrm{Cys} 112 \mathrm{Arg})$ & 0.0030 \\
\hline IL6 & $-572 \mathrm{G} \rightarrow \mathrm{C}$ & 0.0118 & $F A B P 2$ & 2445G $\rightarrow \mathrm{A}(\mathrm{Ala54Thr})$ & 0.0035 \\
\hline SELE & $561 \mathrm{~A} \rightarrow \mathrm{C}(\operatorname{Ser} 128 \mathrm{Arg})$ & 0.0133 & TNFSF4 & $\mathrm{A} \rightarrow \mathrm{G}$ & 0.0043 \\
\hline$S A H$ & $\mathrm{~A} \rightarrow \mathrm{G}$ in intron 12 & 0.0151 & $A P O E$ & 4070C $\rightarrow \mathrm{T}(\operatorname{Arg} 158 \mathrm{Cys})$ & 0.0196 \\
\hline ANXA5 & $-1 \mathrm{C} \rightarrow \mathrm{T}$ & 0.0174 & $A B C C 8$ & $\mathrm{G} \rightarrow \mathrm{A}(\operatorname{Arg} 1273 \mathrm{Arg})$ & 0.0211 \\
\hline COL3Al & $\mathrm{A} \rightarrow \mathrm{G}(\mathrm{Ile} 1205 \mathrm{Val})$ & 0.0265 & $G C K$ & $-30 \mathrm{G} \rightarrow \mathrm{A}$ & 0.0212 \\
\hline$A B C A 1$ & $2583 \mathrm{~A} \rightarrow \mathrm{G}($ Ile823Met $)$ & 0.0294 & COMT & $\mathrm{G} \rightarrow \mathrm{A}($ Val158Met $)$ & 0.0237 \\
\hline \multirow[t]{7}{*}{ COL3Al } & $\mathrm{G} \rightarrow \mathrm{A}(\mathrm{Ala698Thr})$ & 0.0474 & MTHFR & $677 \mathrm{C} \rightarrow \mathrm{T}(\mathrm{Ala} 222 \mathrm{Val})$ & 0.0239 \\
\hline & & & АРOH & 341G $\rightarrow$ A (Ser88Asn) & 0.0254 \\
\hline & & & $E N G$ & $\mathrm{C} \rightarrow \mathrm{G}$ (Asp366His) & 0.0271 \\
\hline & & & $P 2 R Y 12$ & $744 \mathrm{~T} \rightarrow \mathrm{C}$ & 0.0377 \\
\hline & & & $G P 1 B A$ & $1018 \mathrm{C} \rightarrow \mathrm{T}(\mathrm{Thr} 145 \mathrm{Met})$ & 0.0415 \\
\hline & & & GYS1 & $\mathrm{A} \rightarrow \mathrm{G}(\mathrm{Met} 416 \mathrm{Val})$ & 0.0422 \\
\hline & & & $M M P 2$ & $-1306 \mathrm{C} \rightarrow \mathrm{T}$ & 0.0466 \\
\hline
\end{tabular}

Supplementary Table VII. Multivariable logistic regression analysis of polymorphisms related to ACI in nondiabetic individuals.

\begin{tabular}{|c|c|c|c|c|c|c|c|c|c|}
\hline \multirow[b]{2}{*}{ Gene } & \multirow[b]{2}{*}{ Polymorphism } & \multicolumn{2}{|r|}{ Dominant } & \multicolumn{2}{|r|}{ Recessive } & \multicolumn{2}{|c|}{ Additive 1} & \multicolumn{2}{|c|}{ Additive 2} \\
\hline & & $\mathrm{P}$ & OR $(95 \% \mathrm{CI})$ & $\mathrm{P}$ & OR $(95 \% \mathrm{CI})$ & $\mathrm{P}$ & OR $(95 \% \mathrm{CI})$ & $\mathrm{P}$ & OR $(95 \% \mathrm{CI})$ \\
\hline$M M P 12$ & $-82 \mathrm{~A} \rightarrow \mathrm{G}$ & 0.0052 & $2.05(1.22-3.36)$ & & & 0.0052 & $2.05(1.22-3.36)$ & & \\
\hline IL6 & $-572 \mathrm{G} \rightarrow \mathrm{C}$ & 0.0854 & & 0.0066 & $1.40(1.10-1.78)$ & 0.2962 & & 0.0412 & $1.86(1.06-3.51)$ \\
\hline SELE & $561 \mathrm{~A} \rightarrow \mathrm{C}($ Ser128Arg $)$ & 0.0393 & $1.57(1.01-2.38)$ & 0.1465 & & 0.0760 & & 0.1415 & \\
\hline$S A H$ & $\mathrm{~A} \rightarrow \mathrm{G}$ in intron 12 & 0.0364 & $0.43(0.18-0.89)$ & 0.8618 & & 0.0380 & $0.43(0.18-0.89)$ & 0.8607 & \\
\hline ANXA5 & $-1 \mathrm{C} \rightarrow \mathrm{T}$ & 0.3737 & & 0.7237 & & 0.6536 & & 0.7233 & \\
\hline COL $3 A 1$ & $\mathrm{~A} \rightarrow \mathrm{G}(\mathrm{Ile} 1205 \mathrm{Val})$ & 0.1596 & & 0.6908 & & 0.2372 & & 0.6894 & \\
\hline$A B C A 1$ & $2583 \mathrm{~A} \rightarrow \mathrm{G}(\mathrm{Ile} 823 \mathrm{Met})$ & 0.4747 & & 0.0256 & $1.31(1.04-1.66)$ & 0.1069 & & 0.6835 & \\
\hline COL3A1 & $\mathrm{G} \rightarrow \mathrm{A}(\mathrm{Ala698Thr})$ & 0.1379 & & 0.0108 & $1.65(1.11-2.40)$ & 0.4701 & & 0.0081 & $1.71(1.14-2.53)$ \\
\hline
\end{tabular}

OR, odds ratio; CI, confidence interval. Multivariable logistic regression analysis was performed with adjustment for age, sex, BMI, and the prevalence of smoking, hypertension, and hypercholesterolemia. 